\title{
Anurofauna da Colônia Castelhanos, na Área de Proteção Ambiental de Guaratuba, Serra do Mar paranaense, Brasil
}

\author{
Adriele Karlokoski Cunha ${ }^{1 *}$ \\ Igor Soares de Oliveira ${ }^{1}$ \\ Marilia Teresinha Hartmann² \\ ${ }^{1}$ Laboratório de Citogenética Animal, Departamento de Genética, \\ Setor de Ciências Biológicas Universidade Federal do Paraná - UFPR, CEP 81531-990, Curitiba - PR, Brasil \\ ${ }^{2}$ Universidade Federal do Pampa - UNIPAMPA, São Gabriel - RS, Brasil \\ *Autor para correspondência \\ adriele_cunha@yahoo.com.br
}

Submetido em 24/09/2009

Aceito para publicação em 15/03/2010

\section{Resumo}

A riqueza de espécies e a distribuição espacial dos anuros foram estudadas ao longo de 12 meses em uma região de Floresta Atlântica no Município de São José dos Pinhais, estado do Paraná, Brasil. Foram registradas 32 espécies, distribuídas em dez famílias: Brachycephalidae (2), Bufonidae (2), Centrolenidae (1), Cycloramphidae (1), Hemiphractidae (1), Hylidae (18), Hylodidae (1), Leiuperidae (2), Leptodactylidae (3) e Microhylidae (1). A área aberta apresentou 16 espécies, enquanto a área de borda apresentou 17 e o interior de floresta 20. Quanto à utilização dos microhabitats, as espécies foram encontradas: 1) no solo (oito); 2) na água (duas); 3) estrato inferior (onze); 4) estrato intermediário (cinco); 5) estrato superior (quatro). Cinco foram abundantes (15,6\%), doze foram comuns $(37,5 \%)$ e quinze foram raras $(46,9 \%)$. A maioria das espécies é característica de áreas florestais, reforçando a importância da conservação da Floresta Atlântica.

Unitermos: anuros, inventário, Floresta Atlântica

\section{Abstract}

Species richness and structure of an anuran community in an Atlantic Forest site in southern Brazil. The species richness and spatial distribution of an anuran community were studied over 12 months in an Atlantic Forest area in São José dos Pinhais Municipality, Paraná State, southern Brazil. During field surveys, we registered 32 species from ten families: Brachycephalidae (2), Bufonidae (2), Centrolenidae (1), Cycloramphidae (1), Hemiphractidae (1), Hylidae (18), Hylodidae (1), Leiuperidae (2), Leptodactylidae (3), and Microhylidae (1). Sixteen species were registered in open areas, while seventeen species were found on forest borders and twenty species in forest areas. In relation to the microhabitat utilization, species were registered according to stratum of vocalization: 1) on the ground (eight); 2) in the water (two); 3) in the lower stratum (eleven); 4) in the intermediate stratum (five); 5) in the upper stratum (four). Five species were abundant (15.6\%), while twelve were common (37.5\%), and fifteen were considered rare (46.9\%). The biological aspects of the majority of the species described in this work as related to forest areas are not well known. This fact reinforces the importance of Atlantic Forest conservation.

Key words: anurans, Atlantic Forest, inventory 


\section{Introdução}

A obtenção de listas de espécies constitui o primeiro passo para o monitoramento da fauna e da flora em determinadas regiões, pois contribuem para o conhecimento de habitats, avaliação do estado de conservação de espécies e servem como ferramenta auxiliar para medidas de conservação (Pimenta et al., 2005). A falta de conhecimento sobre a diversidade, riqueza, composição das assembléias, distribuição geográfica, relações ecológicas e evolutivas das espécies nativas de anfíbios anuros é um fator limitante para o planejamento e tomada de decisões sobre estratégias de conservação destes animais (Garcia e Vinciprova, 2003; Silvano e Segalla, 2005).

Várias espécies de anfíbios da Floresta Atlântica são pouco conhecidas quanto à taxonomia, história natural e ecologia, devido ao número reduzido de estudos a médio e longo prazo e à falta de levantamentos faunísticos em diferentes regiões (Haddad e Sazima, 1992; Pombal e Gordo, 2004). Junto a essa falta de conhecimento, soma-se o fato de que a Mata Atlântica é um dos ecossistemas mais devastados e mais seriamente ameaçados do Brasil (Meyers et al., 2000). Os remanescentes mais significativos desse bioma estão na região sul e sudeste, principalmente no litoral do estado do Paraná e no sul do estado de São Paulo onde se encontra a maior área contínua de remanescentes da Mata Atlântica (Câmara, 2005).

Atualmente são conhecidas cerca de 120 espécies de anfíbios anuros no Paraná: Bernarde e Anjos (1999), Bernarde e Machado (2000), Castanho (2000), Lingnau e Bastos (2003), Conte (2004), Machado (2004), Segalla e Langone (2004), Conte e Machado (2005), Conte e Rossa-Feres (2006), Garey (2007). No entanto, a riqueza da anurofauna paranaense é ainda maior, devido a registros recentes (Conte et al., 2009) e à descrição de novas espécies (e.g. Ribeiro et al., 2005; Alves et al., 2006; Steinbach-Padilha, 2008) ocorrentes no estado. A fauna paranaense apresenta uma riqueza que reflete a diversidade de biomas e ecossistemas presentes no estado e de acordo com o Livro Vermelho de Espécies ameaçadas do Paraná, 25 espécies de anfíbios estão ameaçadas em algum grau (Mikich e Bérnils, 2004). No entanto, os anfíbios do Paraná são pouco estudados e existem estimativas que cerca de $10 \%$ ainda não tenham sido descritos (Mikich e Bérnils, 2004).

O presente estudo teve por objetivo fornecer uma listagem de espécies de anuros de uma área de Floresta Atlântica paranaense, na Área de Proteção Ambiental (APA) de Guaratuba na Serra do Mar, apresentando dados sobre o ambiente em que foram encontradas e destacando espécies raras, comuns e abundantes na área.

\section{Material e Métodos}

\section{Área de estudo}

O estudo foi desenvolvido na Colônia Castelhanos (Figura 1), localizada na Área de Proteção Ambiental (APA) de Guaratuba na Serra do Mar, estado do Paraná (2547,968'S, 48 54,664'O). A maior área de Castelhanos é composta por Floresta Ombrófila Densa (FOD) Submontana (Roderjan et al., 2002) com altitude variando entre 280 e $550 \mathrm{~m}$ e clima temperado quente (Cfa de Köppen).

Visando facilitar a compreensão da distribuição da anurofauna, a área de estudo foi dividida em três ambientes: área aberta, área de borda e área de floresta. Foram amostrados dois pontos em cada um desses ambientes: ponto 1 e 2, área aberta; 3 e 4, área de borda; e 5 e 6, área de floresta. Área aberta: Ponto $1-$ ANTROPIZADO (ANT) $\left(25^{\circ} 47,968^{\prime} \mathrm{S}, 48^{\circ} 54,664^{\prime} \mathrm{O}\right)$ área alterada, apresentando pequenas edificações próximas a pastagens, pequena plantação de canade-açúcar e criação de aves (patos e galinhas); Ponto 2 - PASTO (PAS) $\left(25^{\circ} 47,7 \mathrm{~S}, 48^{\circ} 54,7 \mathrm{O}-282 \mathrm{~m}\right)$ : área de pastagem com três poças temporárias e banhados próximos à margem direita de rio (Rio Castelhanos). Área de borda: Ponto 3 -BOSQUE (BOS) $\left(25^{\circ} 47,892^{\prime}\right.$ 'S, $\left.48^{\circ} 54,664^{\prime} \mathrm{O}\right)$ : bosque com predominância de palmeiras (Euterpe edulis) nativas e com presença de Pinus sp. A área é sombreada em tempo integral, com vegetação pouco densa e dossel elevado, limitada pelo Rio Castelhanos, moradias e um pequeno córrego; Ponto 4 - CILIAR (CIL) $\left(25^{\circ} 47,709^{\prime} \mathrm{S}, 48^{\circ} 54,713^{\prime} \mathrm{O}\right)$ margem esquerda do Rio Castelhanos, formada por vegetação ciliar bem preservada. Área de floresta: Ponto 5 FLORESTA (FLO) $\left(25^{\circ} 47,027^{\prime} \mathrm{S}, 48^{\circ} 54,504^{\prime} \mathrm{O}\right)$ extensa 


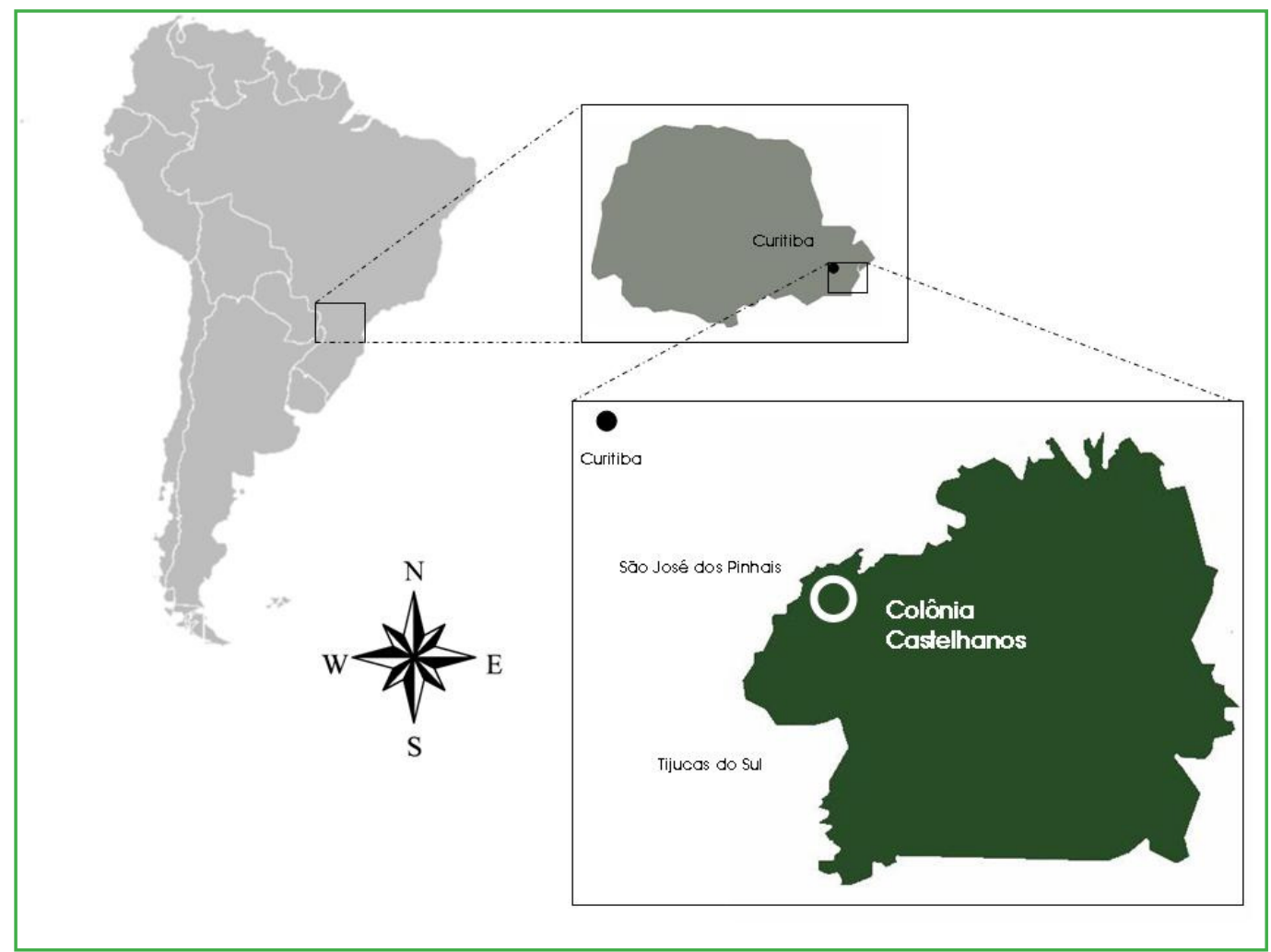

FIGURA 1: Localização geográfica da Colônia Castelhanos no município de São José dos Pinhais na Área de Proteção Ambiental de Guaratuba.

floresta primária ( $4 \mathrm{~km}$ de trilha percorrida), porém com duas áreas pequenas de bananal e terreno muito acidentado. Altitude variando entre $300 \mathrm{~m}$ e $515 \mathrm{~m}$. Ponto 6 - RIO (2546,723’ S, 48 $54,352^{\prime}$ O) córrego em meio à floresta primária. Fundo arenoso com corredeiras e margens sombreadas com dossel elevado.

\section{Amostragens e análises}

Foram realizadas fases mensais regulares, entre maio de 2006 e abril de 2007, com duas incursões ao campo nos meses de novembro, dezembro, janeiro e fevereiro, totalizando 16 visitas. Em outubro e dezembro de 2007, janeiro de 2008 e dezembro de 2009, foram realizadas visitas adicionais (uma em cada mês) que auxiliaram no inventário da anurofauna. Sendo assim, o número total de visitas ao local de estudo passou para 20 , considerando-se as fases regulares e adicionais.

Cada fase teve duração de dois a cinco dias e todos os ambientes foram vistoriados no período diurno e noturno, num total de $100 \mathrm{~h}$ noturnas e $88 \mathrm{~h}$ diurnas. A fase adicional de dezembro de 2009 teve duração de um dia e foi considerada no presente estudo apenas para efeito de composição da lista específica, dado o novo registro fornecido para a área estudada.

Para o registro das espécies foram utilizados os seguintes métodos: busca ativa (Marques e Sazima, 2004), amostragem em sítio de reprodução (sensu Scott e Woodward, 1994) e armadilhas de interceptação e queda (e.g. Cechin e Martins, 2000). Os registros dos indivíduos adultos foram realizados por reconhecimento da vocalização emitida pelos machos ou por sua visualização. Durante o dia os anuros foram procurados sob troncos, pedras ou na serapilheira.

Uma linha de armadilhas de interceptação e queda foi instalada na área de borda, na região de mata ciliar em um transecto com três baldes de $60 \mathrm{~L}$ com intervalo de seis metros entre eles. O estado extremamente acidentado do terreno e a composição argilosa ou rochosa do solo inviabilizaram a instalação de mais armadilhas em outros ambientes da área. Uma cerca-guia de um metro 
de altura foi instalada entre os baldes. A armadilha foi aberta no início e fechada no final de cada visita, sendo vistoriada diariamente.

Em cada local amostrado, os microhabitats utilizados pelos machos para vocalização foram registrados. A altura do substrato ou poleiro em que machos cantores vocalizaram foi medida e foram determinadas categorias de estratificação da seguinte forma: estrato inferior (machos vocalizando em até 50 $\mathrm{cm}$ de altura); estrato intermediário (machos cantores empoleirados acima de $50 \mathrm{~cm}$ e abaixo de $150 \mathrm{~cm}$ de altura) e estrato superior (machos vocalizando acima de $150 \mathrm{~cm}$ ).

Os modos reprodutivos das espécies foram determinados por observações diretas durante as amostragens, com base na literatura (e.g. Dixo e Verdade, 2006; Garey, 2007; Miranda et al., 2008) e definidos de acordo com Haddad e Prado (2005).

As espécies encontradas foram classificadas segundo o índice de Constância de Ocorrência (Dajoz, 2005), onde as espécies foram consideradas: abundantes, presentes em mais de 50\% dos levantamentos; espécies comuns, presentes entre 25 e $50 \%$ das amostragens; espécies raras, presentes em menos de $25 \%$ dos levantamentos.

Foi construída a curva do coletor com 100 aleatorizações para cada ambiente amostrado com o auxílio do programa EstimateS 8.2.0 (Colwell, 2006). Para esta análise foram consideradas somente as espécies registradas durante o período regular de estudo.

Exemplares testemunhos foram depositados na coleção herpetológica do Museu de Ciências Naturais da Universidade Federal do Paraná (MCNUFPR). A coleção herpetológica do Museu de História Natural Capão da Imbuia (MHNCI) foi consultada para a elaboração da lista de anfíbios de Castelhanos, todavia não foram encontrados dados referentes à localidade estudada.

\section{Resultados}

Foi registrado um total de 32 espécies de anfíbios anuros pertencentes a dez famílias e dezessete gêneros:
Brachycephalidae (duas), Bufonidae (três), Centrolenidae (uma), Cycloramphidae (uma), Hemiphractidae (uma), Hylidae (18), Hylodidae (uma), Leiuperidae (duas), Leptodactylidae (três) e Microhylidae (uma) (Tabela 1 e Figura 2). Dentre estas, Dendropsophus nahdereri foi encontrada somente na fase adicional de outubro e dezembro de 2007, Chiasmocleis leucosticta em janeiro de 2008 e Hypsiboas albomarginatus em dezembro de 2009 .

Cerca de $90 \%$ da anurofauna foi registrada durante as visitas aos sítios de vocalização (Tabela 1). A busca ativa possibilitou o registro de $55 \%$ das espécies e duas (Trachycephalus mesophaeus e Scinax gr. catharinae) foram registradas somente por este método. Seis espécies (20,7\%) foram amostradas nas armadilhas de interceptação e queda e Brachycephalus aff. hermogenesi foi registrada exclusivamente por este método.

A curva do coletor para a área aberta (Figura 3A) mostrou estabilização em assíntota, na qual a riqueza estimada foi 15, enquanto que para a área de borda florestal (Figura 3B), não houve a formação da assíntota e a riqueza estimada foi $17 \pm 2,5$ espécies. Para o interior de floresta (Figura 3C) a curva apresentou uma tendência à estabilização, com riqueza estimada de 19 $\pm 0,94$ espécie.

Os ambientes apresentaram composição semelhante de espécies: 16 na área aberta, 17 na borda e 20 na floresta, porém o ponto de amostragem com maior riqueza foi floresta (FLO), com 20 espécies, representando cerca de $64 \%$ do que foi registrado neste estudo. O ponto na área antropizada (ANT) foi o que apresentou menos espécies, com 9,7\% das espécies registradas, seguido do ponto RIO com 12,9\% (Tabela 1). Com relação à composição taxonômica, sete espécies foram registradas tanto em área de borda quanto em área de floresta (Bokermannohyla hylax, Flectonotus sp., Vitreorana uranoscopa, Hylodes heyeri, Ischnocnema aff. guentheri, Leptodactylus cf. marmoratus e Rhinella abei). Seis espécies foram encontradas somente no interior de floresta (Aplastodiscus albosignatus, Chiasmocleis leucosticta, Phyllomedusa distincta, Physalaemus maculiventris, Scinax gr. catharinae e $S$. rizibilis) e seis somente na área aberta (Dendropsophus berthalutzae, D. elegans, D. 
nahdereri, D. werneri, Hypsiboas faber e Scinax alter). Cinco espécies estiveram presentes nos três ambientes simultaneamente (Hypsiboas bischoffi, Leptodactylus notoaktites, Physalaemus olfersii, Proceratophrys boiei e Scinax perereca). Três táxons foram encontrados tanto em borda quanto em área aberta (Dendropsophus minutus, Leptodactylus cf. latrans e Rhinella icterica), dois em ambientes abertos e florestais (Dendropsophus microps e Scinax fuscovarius) e dois exclusivamente na borda da mata (Brachycephalus aff. hermogenesi e Trachycephalus mesophaeus).
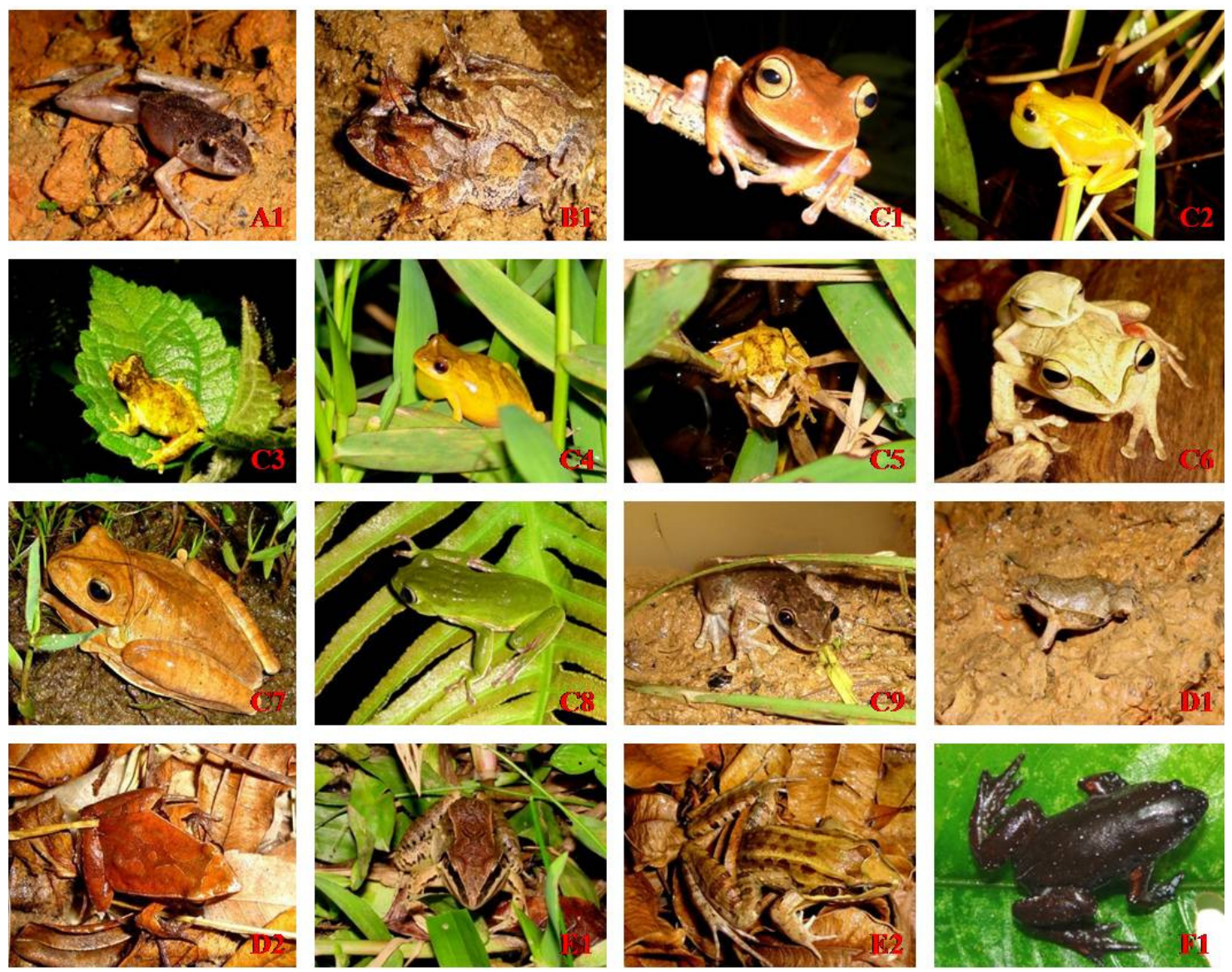

FIGURA 2: Algumas famílias e espécies de anuros registradas na Colônia Castelhanos, Paraná entre maio de 2006 e abril de 2007: A - Brachycephalidae (A1 - Ischnocnema aff. guentheri); B - Cycloramphidae (B1 - Proceratophrys boiei); C - Hylidae (C1 Bokermannohyla hylax, C2 - Dendropsophus elegans, C3 - D. microps, C4 - D. minutus, C5 - D. werneri, C6 - Hypsiboas bischoffi, C7 - H. faber, C8 - Phyllomedusa distincta, C9 - Scinax fuscovarius); D - Leiuperidae (D1 - Physalaemus maculiventris, D2 - P. olfersii); E - Leptodactylidae (E1 - Leptodactylus notoaktites, E2 - Leptodactylus cf. latrans); F Microhylidae (F1 - Chiasmocleis leucosticta). Fotos: I. S. Oliveira. 




FIGURA 3: Curvas de acumulação de espécies calculadas a partir da ocorrência por amostra durante o período regular de estudo (maio de 2006 a abril de 2007) para: A - área aberta; B - área de borda e C - interior de floresta. Os dados foram aleatorizados 100 vezes em cada curva no programa EstimateS versão 8.2.0. (Colwell, 2006) As linhas acima e abaixo correspondem ao intervalo de confiança $(95 \%)$.

TABELA 1: Famílias e espécies de anuros registradas na Colônia Castelhanos, Paraná, no período regular do estudo, entre maio de 2006 e abril de 2007 com informações sobre: método de amostragem (1 - busca ativa, 2 - sítio de vocalização, 3 - armadilhas $)$; constância de ocorrência - CO (C - comum, $\mathrm{R}$ - rara, A abundante); ambiente de encontro (área aberta, borda e floresta); ponto de amostra (ANT, PAS, BOS, CIL, FLO, RIO); modo reprodutivo (sensu Haddad e Prado, 2005), sendo CB relativo à informação obtida por consulta bibliográfica e OP por observação pessoal; e ocorrência das espécies por ambiente, com + representando presença; - ausência; \# dados não avaliados e * registros em visitas posteriores ao período regular de estudo.

\begin{tabular}{|c|c|c|c|c|c|c|c|c|c|}
\hline \multirow{2}{*}{ Famílias e Espécies } & \multirow[b]{2}{*}{ Método } & \multirow[b]{2}{*}{$\mathrm{CO}$} & \multicolumn{2}{|c|}{ Aberta } & \multicolumn{2}{|c|}{ Borda } & \multicolumn{2}{|c|}{ Floresta } & \multirow[b]{2}{*}{ Modo } \\
\hline & & & ANT & PAS & BOS & CIL & FLO & RIO & \\
\hline \multicolumn{10}{|l|}{ Brachycephalidae } \\
\hline Brachycephalus aff. hermogenesi & 3 & $\mathrm{R}$ & - & - & - & + & - & - & $23_{\mathrm{CB}}$ \\
\hline Ischnocnema aff. guentheri & 1,2 & A & - & - & + & + & + & - & $23_{\mathrm{CB}}$ \\
\hline \multicolumn{10}{|l|}{ Bufonidae } \\
\hline $\begin{array}{l}\text { Rhinella abei (Baldissera, Caramaschi \& Haddad, } \\
\text { 2004) }\end{array}$ & $1,2,3$ & $\mathrm{R}$ & - & - & - & + & + & - & $1_{\mathrm{CB}}$ \\
\hline Rhinella icterica (Spix, 1824) & $1,2,3$ & $\mathrm{R}$ & + & - & - & + & - & - & $1_{\mathrm{CB}}$ \\
\hline \multicolumn{10}{|l|}{ Centrolenidae } \\
\hline Vitreorana uranoscopa (Müller, 1924) & 2 & $\mathrm{C}$ & - & - & + & + & - & + & $25_{\mathrm{CB}}$ \\
\hline \multicolumn{10}{|l|}{ Cycloramphidae } \\
\hline Proceratophrys boiei (Wied-Neuwied, 1824) & $1,2,3$ & $\mathrm{C}$ & + & - & - & + & + & - & $1_{\mathrm{CB}}$ \\
\hline
\end{tabular}




\section{Hemiphractidae}

Flectonotus sp.

\section{Hylidae}

Aplastodiscus albosignatus (Lutz \& Lutz, 1938)

Bokermannohyla hylax (Heyer, 1985)

Dendropsophus berthalutzae (Bokermann, 1962)

Dendropsophus elegans (Wied-Neuwied, 1824) 2

Dendropsophus microps (Peters, 1872)

Dendropsophus minutus (Peters, 1872)

*Dendropsophus nahdereri (Lutz \& Bokermann, 1963)

Dendropsophus werneri (Cochran, 1952)

Hypsiboas bischoffi (Boulenger, 1887)

Hypsiboas faber (Wied-Neuwied, 1821)

*Hypsiboas albomarginatus (Spix, 1824)

Phyllomedusa distincta Lutz, 1950

Scinax alter (Lutz, 1973)

Scinax gr. catharinae

Scinax fuscovarius (Lutz, 1925)

Scinax perereca Pombal, Haddad \& Kasahara, 1995

Scinax rizibilis (Bokermann, 1964)

Trachycephalus mesophaeus (Hensel, 1867)

\section{Hylodidae}

Hylodes heyeri Haddad, Pombal \& Bastos, 1996

\section{Leiuperidae}

Physalaemus maculiventris (Lutz, 1925)

Physalaemus olfersii (Lichtenstein \& Martens, 1856)

\section{Leptodactylidae}

Leptodactylus cf. marmoratus (Steindachner, 1867)

Leptodactylus notoaktites Heyer, 1978

Leptodactylus cf. latrans (Steffen, 1815)

\section{Microhylidae}

*Chiasmocleis leucosticta (Boulenger, 1888)
2

1,2

2

2

2

\#

2

1,2

1,2

\#

2

2

1

1,2

1,2

2

1

2

1,2

$1,2,3$

1,2

1,2

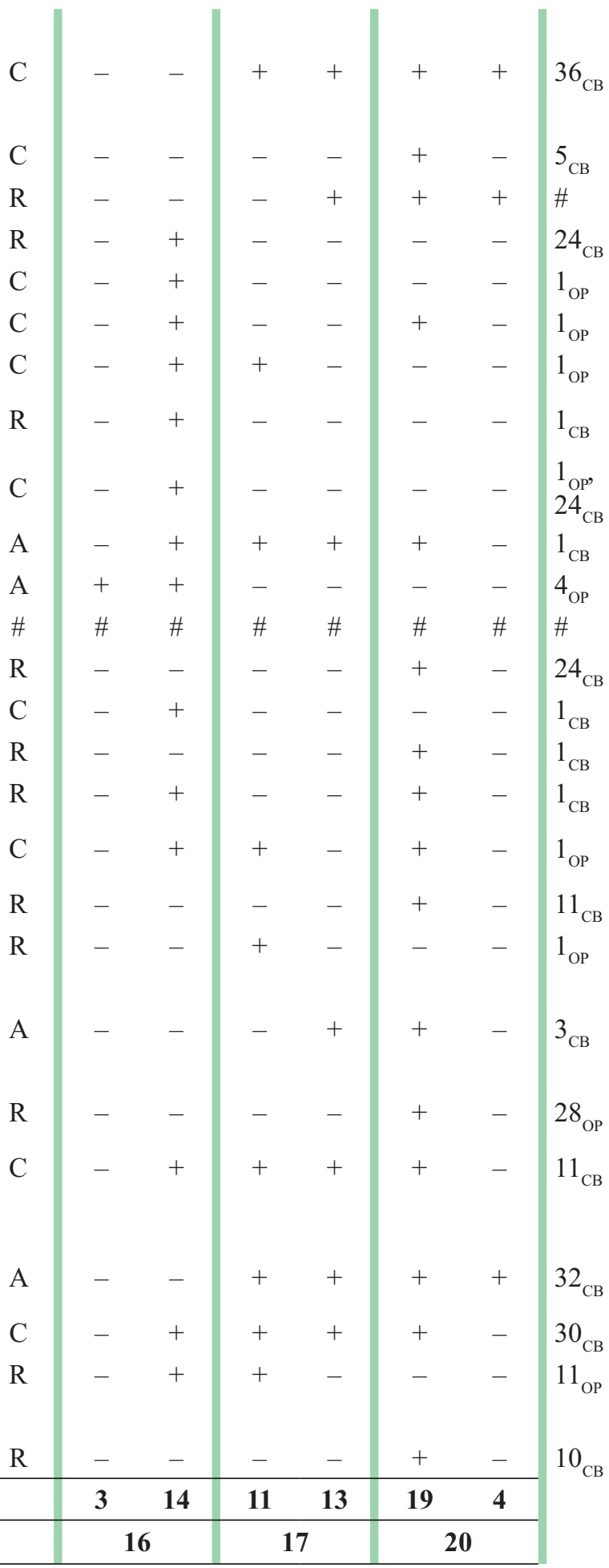

Quanto à utilização dos microhabitats, as espécies foram encontradas: 1) no solo (machos vocalizando no solo úmido): Hypsiboas faber, Ischnocnema aff. guentheri, Leptodactylus cf. marmoratus, L. notoaktites, Physalaemus olfersii, Proceratophrys boiei, Rhinella abei e R. icterica; 2) na água (os machos vocalizam dentro da água, com parte do corpo submerso): Physalaemus maculiventris e P. olfersii; 3) na vegetação: estrato inferior: Dendropsophus berthalutzae, D. elegans, D. microps, D. minutus, D. 
werneri, Hylodes heyeri, Ischnocnema aff. guentheri, Scinax alter, S. fuscovarius, S. perereca e S. rizibilis; 4) estrato intermediário: Bokermannohyla hylax, Hypsiboas bischoffi, Ischnocnema aff. guentheri, Leptodactylus cf. marmoratus e Phyllomedusa distincta; 5) estrato superior: Aplastodiscus albosignatus, Bokermannohyla hylax, Flectonotus sp. e Vitreorana uranoscopa.

Em relação à constância de ocorrência das espécies no ambiente, cinco foram abundantes (15,6\%), 12 foram comuns $(37,5 \%)$ e 15 foram raras $(46,9 \%)$. A área com maior número de espécies consideradas de ocorrência rara foi a floresta (ponto FLO), com oito espécies. A borda teve mais espécies comuns (sete), enquanto as abundantes ocorreram em mais de um ambiente.

Foram registrados 13 modos reprodutivos para a anurofauna de Castelhanos (sensu Haddad e Prado, 2005). Dentre as espécies, somente Bokermannohyla hylax não possui modo reprodutivo conhecido. A família Hylidae apresentou o maior número de modos reprodutivos (cinco), seguida por Leptodactylidae (três), Leiuperidae (dois) e das demais famílias (um) (Tabela 1). O modo reprodutivo 1, caracterizado como ovos e girinos exotróficos em água lêntica, foi utilizado por 14 espécies que ocorreram em áreas abertas, borda e interior de floresta. Além deste, o modo 11 (ninhos de espuma flutuantes em poças com girinos exotróficos) e o modo 30 (ninhos de espuma subterrâneos com girinos exotróficos) também foram característicos das espécies que ocorreram em todos os ambientes. Cinco modos reprodutivos foram registrados para as espécies que utilizaram as bordas e o interior da floresta (i.e. 3 - ovos e desenvolvimento larval em câmaras subaquáticas com girinos exotróficos em riachos, 23 - ovos terrestres sobre o solo com desenvolvimento direto, $25-$ ovos depositados em folhas com girinos exotróficos que caem em ambientes lóticos, 32 - ovos em ninho de espuma em toca subterrânea e girinos com desenvolvimento no interior do ninho, 36 - ovos carregados em bolsa no dorso das fêmeas com girinos endotróficos em bromélias e bambus). O modo 4, qualificado como ovos depositados em bacias com girinos exotróficos em poças ou riachos foi exclusivo de Hypsiboas faber que ocorreu somente em área aberta. Os modos 5 (ovos e desenvolvimento larval em ninhos subterrâneos, com girinos exotróficos em poças ou riachos), 10 (ninho de bolha flutuante em poças com girinos exotróficos) e 28 (ninho de espuma no chão de floresta com girinos exotróficos em poças) são pertencentes à Chiasmocleis leucosticta, Aplastodiscus albosignatus e Physalaemus maculiventris, respectivamente; espécies que foram encontradas somente em ambiente florestal. $O$ modo 24 (i.e. desova depositada na vegetação marginal, com girinos exotróficos que caem na água após eclosão), foi característico de Dendropsophus berthalutzae, D. werneri (espécies de área aberta) e Phyllomedusa distincta (espécie de floresta). Dendropsophus werneri pode apresentar os modos reprodutivos 1 e 24 (e.g. Garey, 2007; Miranda et al., 2008) porém em Castelhanos, só o modo 1 foi observado para a espécie.

\section{Discussão}

Considerando que o Paraná tem em torno de 128 espécies de anuros registradas (Segalla e Langone, 2004; Ribeiro et al., 2005; Alves et al., 2006, SteinbachPadilha, 2008; Conte et al., 2009), a riqueza encontrada na área corresponde a $25 \%$ da riqueza total do estado. A heterogeneidade ambiental tem sido reconhecida como uma das melhores explicações para a variação na diversidade de espécies (Huston, 1994). Diversos estudos desenvolvidos no Brasil (e.g. Cardoso et al., 1989; Pombal, 1997; Bernarde e Kokubum, 1999) sugerem que ambientes complexos permitem a coexistência de um número maior de espécies de anuros que ambientes homogêneos, por disponibilizar maior número de microambientes. A área estudada apresenta essa heterogeneidade, com áreas abertas, áreas de borda e floresta fechada, cada uma com características exclusivas de luminosidade, umidade e tipo de vegetação e com colonização por diferentes espécies de anuros.

O número de espécies encontrado é um reflexo do uso de mais de um método de levantamento. Visita a sítios de reprodução, busca ativa e armadilhas de interceptação e queda são métodos complementares e eficientes em inventários de anurofauna (e.g. Conte e Rossa-Feres, 2006), uma vez que cada método utilizado neste estudo forneceu registros exclusivos.

Com relação à curva do coletor, os padrões encontrados podem ser relacionados com as características de cada ambiente. Na área aberta, o teste mostra que o 
esforço de coleta foi suficiente para registrar o número de espécies estimado. Originalmente, estas áreas possuíam cobertura florestal e, devido à ocupação humana, o ambiente foi modificado. Haddad e Prado (2005) afirmam que ambientes desmatados podem apresentar redução na riqueza por mudanças microclimáticas. Além disso, a falta de ambientes apropriados para a reprodução de grande parte das espécies de anuros, limita a ocorrência da maioria das espécies (Haddad, 1998; Haddad e Prado, 2005). Na área de borda o teste aponta para insuficiência amostral, indicando que mais espécies podem ocorrer neste ambiente. Este padrão é esperado, uma vez que a borda representa uma área de transição e não possui uma anurofauna característica (e.g. Machado, 2004). A ocorrência de espécies de áreas abertas (e.g. Dendropsophus minutus e Rhinella icterica) na borda florestal reforça a idéia de que a borda constitui uma área de transição, onde espécies generalistas no uso do habitat podem ser eventualmente encontradas, pois podem utilizar o ambiente florestal como abrigo e forrageio (Silva e Rossa-Feres, 2007). Por fim, a curva relativa à floresta tendeu à estabilização, o que indica que o esforço empregado forneceu o registro da maior parte das espécies esperadas, mas que há a probabilidade de novos registros. Este resultado também era esperado, pois novas espécies foram encontradas após o período regular de estudo (e.g. Chiasmocleis leucosticta, Dendropsophus nahdereri e Hypsiboas albomarginatus). Ambientes florestais apresentam maior heterogeneidade e podem sustentar maior riqueza (Williams e Hero, 2001). Castanho (2000) registrou 39 espécies de anuros e Garey (2007) 42; ambos em Guaraqueçaba, região com características similares à floresta de Castelhanos, indicando que muitos outros táxons ainda não amostrados podem ocorrer na área de estudo, o que ressalta a importância de estudos de longa duração com relação à anurofauna.

Flectonotus sp. foi o único táxon cuja identificação além de gênero não foi possível. No Brasil esses anuros ocorrem nas áreas montanhosas da Floresta Atlântica e pouco de sua biologia, distribuição e taxonomia é conhecido, sendo provável que o grupo represente um complexo de espécies (Sinsch e Juraske, 2006; Frost, 2009).
A maior parte das espécies de anuros de Castelhanos foi registrada na borda ou no interior da floresta. Muitas dessas espécies apresentam modos reprodutivos especializados (e.g. Flectonotus sp., Ischnocnema aff. guentheri, Vitreorana uranoscopa) e necessitam de condições específicas - principalmente de umidade - para se reproduzir (Haddad, 1998; Haddad e Prado, 2005). Ambientes florestais podem suprir grande parte dessas necessidades, pois apresentam maior heterogeneidade ambiental (Williams e Hero, 2001; Bertoluci et al., 2007). Por outro lado, o registro em borda de floresta de espécies como Rhinella icterica, Dendropsophus minutus e Leptodactylus cf. latrans, espécies comumente encontradas em áreas abertas (e.g. Heyer et al., 1990; Conte e Machado, 2005), pode estar relacionado com o uso dos ambientes florestais como locais de refúgio, de forrageio, migração, hibernação ou para abrigo de juvenis (e.g. Silva e Rossa-Feres, 2007).

Dois pontos amostrados neste estudo apresentaram baixa riqueza por motivos diferentes. Primeiro, a área aberta antropizada (ANT), com apenas três espécies. Neste caso, a perda de habitats pode ser considerada um fator que diminui a abundância e a diversidade de anfíbios; e a alteração de ambientes representa grande impacto sobre algumas populações de anuros, principalmente em uma área originalmente florestal (Haddad, 1998; Alford e Richards, 1999). Outro fator relevante neste caso é a inexistência de locais adequados à reprodução, como corpos d'água ou serapilheira úmida (Haddad, 1998), visto que são de extrema importância para quase todas as espécies deste estudo (Haddad e Prado, 2005). Outro ponto dentro da área florestada (RIO) teve quatro espécies - somente uma com modo reprodutivo em água corrente. De acordo com Zimmerman e Simberlof (1996), uma possível explicação para o menor número de espécies em RIO é a especialização reprodutiva necessária para ocupar um ambiente de riacho e os fatores históricos da colonização ancestral no Neotrópico. Segundo estes autores, é provável que os ancestrais dos anuros atuais tenham encontrado um ambiente predominantemente lêntico, que proporcionou a irradiação para modos reprodutivos independentes de ambientes lóticos, ao contrário do padrão encontrado em outras regiões do mundo. Das espécies encontradas no ponto RIO, Vitreorana 
uranoscopa tem o modo reprodutivo 25 e seus girinos necessitam de água corrente para se desenvolver (sensu Haddad e Prado, 2005). Bokermannohyla hylax ainda possui modo reprodutivo desconhecido, porém há indícios de que pode utilizar o riacho para o desenvolvimento de girinos (e.g. Garey, 2007). As outras duas espécies (Flectonotus sp. e Leptodactylus marmoratus) são florestais e não dependem da água parada ou corrente para a reprodução (sensu Haddad e Prado, 2005). Essa diferença na utilização do habitat e a necessidade de um ambiente florestal para o modo reprodutivo de algumas espécies é característica da Mata Atlântica (e.g. Haddad e Prado, 2005).

Como o uso de um determinado sítio reprodutivo é característica da história de vida de cada espécie, sendo relacionado com o modo reprodutivo, é esperado que espécies com modos reprodutivos dependentes de uma determinada fisionomia vegetal ou de condições de luminosidade, umidade e temperatura ocorram em locais muito específicos de habitat (e.g. Hartmann, 2004; Haddad e Prado, 2005). Na análise do microhabitat das espécies, a maioria utilizou algum tipo de vegetação: 20 espécies (11 espécies no estrato inferior, cinco no intermediário e quatro no superior). Esses dados de microhabitat também estão diretamente relacionados com a diversidade de modos reprodutivos das espécies da Mata Atlântica, e corroboram as idéias de necessidade de manutenção da floresta, da heterogeneidade ambiental e da diversidade das populações (e.g. Bertoluci e Rodrigues, 2002; Juncá e Borges, 2002; Machado, 2004).

A constância de ocorrência das espécies apontou 15 táxons como raros, sendo a maioria de ambiente florestal, enquanto que as espécies consideradas comuns foram encontradas principalmente na borda. A ocorrência das espécies está relacionada com a atividade sazonal, já que foi levado em conta apenas o registro de aparecimento dos anuros. Várias espécies deste estudo que figuram na categoria rara (e.g. Dendropsophus berthalutzae, D. nahdereri, Leptodactylus cf. latrans, Rhinella abei, R. icterica e Scinax fuscovarius) possuem reprodução prolongada (e.g. Conte e Rossa-Feres, 2006; Garey, 2007; Giasson e Haddad, 2007) e poderiam ter sido registradas a maior parte do ano e, como não o foram, é possível que essas populações sejam pequenas na área estudada (Haddad e Prado, 2005). Se o motivo são flutuações populacionais, modificações ambientais ou o tipo de necessidade de habitat de cada espécie, somente estudos de monitoramento poderiam fornecer essas informações. De qualquer maneira, a remoção de áreas florestais da região pode provocar declínio populacional das espécies que dependem das florestas, visto que o desmatamento da região Neotropical é responsável pela ameaça de extinção de várias espécies de anfíbios (Garcia e Vinciprova, 2003; Segalla e Langone, 2004; Conte e Rossa-Feres, 2007).

Modos reprodutivos são caracterizados como uma combinação de características relacionadas a sítios de oviposição, ovos e desovas, taxas e duração do desenvolvimento, estágio e tamanho da desova e ocorrência de cuidado parental (Salthe e Duellman, 1973). De acordo com a revisão de Haddad e Prado (2005), para a Floresta Atlântica são encontrados 27 modos reprodutivos dos 39 descritos. Para Castelhanos, foram reconhecidos 13 modos reprodutivos. Outras áreas desse bioma apresentaram alta diversidade de modos reprodutivos, como os 14 encontrados por Castanho (2000) e Garey (2007) em Guaraqueçaba (PR), 12 modos por Hartmann (2004) em Ubatuba (SP) e nove modos por Conte (2004) em São José dos Pinhais (PR). A alta diversidade de modos reprodutivos encontrados na Floresta Atlântica pode ser decorrente da utilização de áreas úmidas para a reprodução dos anuros, o que pode indicar partilha espacial do ambiente (Hartmann, 2004). A diversidade dos modos reprodutivos e sua eficiência podem ser importantes para o estabelecimento das espécies de anuros no ambiente (Duellman, 1985), mas também pode representar restrições ao uso de determinados habitats (Bertoluci e Rodrigues, 2002). Neste estudo, o maior número de modos reprodutivos foi encontrado na borda florestal e no interior da floresta, ambientes que potencialmente possuem maior número de microambientes úmidos e maior quantidade de corpos d'água. O modo reprodutivo 1 é considerado o menos especializado (Duellman e Trueb, 1986) e por conta disso é o modo mais comumente encontrado em anuros (e.g. Conte, 2004; Hartmann, 2004; Garey, 2007) e no presente estudo esteve presente em aproximadamente $47 \%$ das espécies. Dendropsophus werneri, pode apresentar dois tipos de modos reprodutivos (1 e 24), o 
que pode estar relacionado com ambientes instáveis e imprevisíveis (Haddad e Pombal, 1998). Em Castelhanos foi registrado pela segunda vez para esta espécie o modo reprodutivo 1 (Miranda et al., 2008).

Este foi o segundo registro de Physalaemus maculiventris e Vitreorana uranoscopa dentro da APA de Guaratuba (Lingnau, 2004) e o primeiro registro de Chiasmocleis leucosticta para a APA. Até onde se sabe essas espécies são sensíveis a alterações do ambiente e informações sobre sua biologia são raras (Segalla e Langone, 2004). Sendo assim, a ocorrência desses táxons em Castelhanos pode indicar que a floresta apresenta ambientes em bom estado de conservação, ressaltando a importância de áreas protegidas.

\section{Agradecimentos}

A Manoela W. Cardoso pelo auxílio em campo e pelas sugestões no trabalho. Ao Reginaldo A. Machado, Rodrigo M. Stuart e ao Mauricio O. Moura pelo auxílio na elaboração do artigo. A Julio C. Moura-Leite pelo acesso à coleção do MHNCI e a Fernando Sedor pelo recebimento dos exemplares no MCNUFPR. Aos colegas de campo Rafael Costa, Kelly Mafra, Talissa Mendes e Marília Higa. Ao Sr. Moacyr Grassmann e a Louri Klemann Jr. Ao IBAMA pela licença concedida e ao SIMEPAR pelas informações climatológicas.

\section{Referências}

Alford, R. A.; Richards, S. J. 1999. Global amphibian declines: a problem in applied ecology. Annual Review of Ecology, Evolution and Systematics, 30: 133-165.

Alves, A. C. R.; Ribeiro, L. F.; Haddad, C. F. B.; Reis, S. F. 2006. Two new species of Brachycephalus (Anura: Brachycephalidae) from the Atlantic Forest in Paraná State, Southern Brazil. Herpetologica, 62 (2): 221-233.

Bernarde, P. S.; Anjos, L. 1999. Distribuição espacial e temporal da anurofauna no Parque Estadual Mata dos Godoy, Londrina, Paraná, Brasil (Amphibia: Anura). Comunicações do Museu de Ciências Tecnológicas da PUCRS, 12: 127-140.

Bernarde, P. S.; Kokubum, M. N. C. 1999. Anurofauna do município de Guararapes, estado de São Paulo, Brasil (Amphibia: Anura). Acta Biológica Leopoldensia, 23 (1): 89-97.

Bernarde, P. S.; Machado, R. A. 2000. Riqueza de espécies, ambientes de reprodução e temporada de vocalização da anurofauna em Três Barras do Paraná, Brasil (Amphibia: Anura). Cuadernos de Herpetologia, 14 (2): 93-104.
Bertoluci, J.; Rodrigues, M. T. 2002. Utilização de habitats reprodutivos e micro-habitats de vocalização em uma taxocenose de anuros (Amphibia) da Mata Atlântica do sudeste do Brasil. Papéis Avulsos de Zoologia, 42 (11): 287-297.

Bertoluci, J.; Brassaloti, R. A.; Ribeiro Jr.; J. W.; Vilela, V. M. F. N.; Sawakuchi, H. O. 2007. Species composition and similarities among anuran assemblages of forest sites in southeastern Brazil. Scientia Agricola, 64 (4): 364-374.

Câmara, I. G. 2005. Breve história da conservação da Mata Atlântica. In: Galindo-Leal, C. \& Câmara, I. G. (Eds). Mata Atlântica: Biodiversidade, ameaças e perspectivas. Conservação Internacional, Belo Horizonte, BR, p.31-42.

Cardoso, A. J.; Andrade, G. V.; Haddad, C. F. B. 1989. Distribuição espacial em comunidade de anfíbios (Anura) no sudeste do Brasil. Revista Brasileira Biologia, 49 (1): 241-249.

Castanho, L. M. 2000. História natural de uma comunidade de anfíbios anuros da região de Guaraqueçaba, litoral norte do estado do Paraná. Tese de Doutorado, Universidade Estadual Paulista, Brasil, 132pp.

Cechin, S. Z.; Martins, M. 2000. Eficiência de armadilhas de queda (pitfall traps) em amostragens de anfíbios e répteis no Brasil. Revista Brasileira de Zoologia, 17: 729-740.

Colwell, R. K. 2006. EstimateS: statistical estimation of species richness and shared species from samples. Version 8. Disponível em $<$ http:purl.oclc.org/estimates $>$. Acesso em 18 de fevereiro de 2010.

Conte, C. E. 2004. Diversidade, distribuição temporal e uso de habitat por anfíbios anuros em São José dos Pinhais, região sudeste do estado do Paraná. Dissertação de Mestrado, Universidade Estadual Paulista, Brasil, 85pp.

Conte, C. E.; Garey, M. V.; Lingnau, R.; Silva, M. X.; Armstrong, C.; Hartmann, M. T. 2009. Amphibia, Anura, Limnomedusa macroglossa, Dendropsophus anceps, D. berthalutzae, D. seniculus, Scinax littoralis: New state records, distribution extension, and filling gaps. Check List, 5 (2): 202-209.

Conte, C. E.; Machado, R. A. 2005. Riqueza de espécies e distribuição espacial e temporal em comunidade de anuros (Amphibia, Anura) em uma localidade de Tijucas do Sul, Paraná, Brasil. Revista Brasileira de Zoologia, 22 (4): 940-948.

Conte, C. E.; Rossa-Feres, D. C. 2006. Diversidade e ocorrência da anurofauna (Amphibia, Anura) em São José dos Pinhais, Paraná, Brasil. Revista Brasileira de Zoologia, 23 (1): 162-175.

Conte, C. E.; Rossa-Feres, D. C. 2007. Riqueza e distribuição espaço-temporal de anuros em um remanescente de Floresta de Araucária no sudeste do Paraná. Revista Brasileira de Zoologia, 24 (4): 1025-1037.

Dajoz, R. 2005. Princípios de Ecologia. $7^{\mathrm{a}}$ ed. Artmed, Porto Alegre, BR, 519pp.

Dixo, M.; Verdade, V. K. 2006. Herpetofauna de serapilheira da Reserva Florestal de Morro Grande, Cotia (SP). Biota Neotropica, 6 (2): 1-20.

Duellman, W. E. 1985. Reproductive modes in anuran amphibians: phylogenetic significance of adaptive strategies. South African Journal of Science, 81: 174-178.

Duellman, W. E.; Trueb, L. 1986. Biology of amphibians. McGrawHill, New York, USA, 650pp. 
Frost, D. R. 2009. Amphibian species of the world: an online reference. Version 5.3 (12 February, 2009). American Museum of Natural History, New York, USA. Disponível em $<$ http:research.amnh. org/herpetology/amphibian/>. Acesso em 23 de Fevereiro de 2010.

Garcia, P. C. A.; Vinciprova, G. 2003. Anfíbios. In: Fontana, C. S.; Bencke, G. A. \& Reis, R. E. (Eds). Livro vermelho da fauna ameaçada de extinção no Rio Grande do Sul. EDIPUCRS, Porto Alegre, BR, p.85-100.

Garey, M. V. 2007. Diversidade de anfíbios anuros em três diferentes estádios sucessionais da Floresta Atlântica da Reserva Natural Salto Morato, Guaraqueçaba - PR. Dissertação de Mestrado, Universidade Federal do Paraná, Brasil, 83pp.

Giasson, L. O. M.; Haddad, C. F. B. 2007. Mate choice and reproductive biology of Hypsiboas albomarginatus (Anura: Hylidae) in the Atlantic Forest, southeastern Brazil. South American Journal of Herpetology, 2: 157-164.

Haddad, C. F. B. 1998. Biodiversidade dos anfíbios do Estado de São Paulo. In: Castro, R. M. C. (Ed). Biodiversidade do estado de São Paulo, Brasil: síntese do conhecimento ao final do século XX. Editora FAPESP, São Paulo, BR, p. 17-26.

Haddad, C. F. B.; Pombal Jr., J. P. 1998. Redescription of Physalaemus spiniger (Anura: Leptodactylidae) and description of two new reproductive modes. Journal of Herpetology, 32 (4): 473-488.

Haddad, C. F. B.; Prado, C. P. A. 2005. Reproductive modes in frogs and their unexpected diversity in the Atlantic Forest of Brazil. BioScience, 55: 207-217.

Haddad, C. F. B.; Sazima, I. 1992. Anfíbios anuros da Serra do Japi. In: Morellato, L. P. C. (Ed.). História Natural da Serra do Japi: Ecologia e preservação de uma área florestal no Sudeste do Brasil. UNICAMP, Campinas, BR, p.188-211.

Hartmann, M. T. 2004. Biologia reprodutiva de uma comunidade de anuros (Amphibia) na Mata Atlântica (Picinguaba, Ubatuba, SP). Tese de Doutorado, Universidade Estadual Paulista, Brasil, 132pp.

Heyer, W. R.; Rand, A. S.; Cruz, C. A. G.; Peixoto, O. L.; Nelson, C. E. 1990. Frogs of Boracéia. Arquivos de Zoologia, 31: 231-410.

Huston, M. A. 1994. Biological diversity - The coexistence of species on changing landscapes. $19^{\text {th }}$ ed. Cambridge University Press, New York, USA, 681pp.

Juncá, F. A.; Borges, C. L. S. 2002. Fauna associada a bromélias terrícolas da Serra da Jibóia, Bahia. Sitientibus Série Ciências Biológicas, 2 (1/2): 73-81.

Lingnau, R. 2004. A importância da "Área de Proteção Ambiental de Guaratuba" para conservação de algumas espécies de anfíbios anuros no estado do Paraná, Brasil. Anais do IV Congresso Brasileiro de Unidades de Conservação, Curitiba, Brasil, p.92.

Lingnau, R.; Bastos, R. P. 2003. Vocalizações de duas espécies de anuros do sul do Brasil (Amphibia: Hylidae). Arquivos do Museu Nacional, 61 (3): 203-207.

Machado, R. A. 2004. Ecologia de assembléias de anfíbios anuros no município de Telêmaco Borba, Paraná, sul do Brasil. Tese de Doutorado, Universidade Federal do Paraná, Brasil, 113pp.

Marques, O. A. V.; Sazima, I. 2004. História natural dos répteis da Estação Ecológica Juréia-Itatins. In: Marques, O. A. V. \& Duleba, W. (Eds). Estação Ecológica Juréia-Itatins: Ambiente físico, flora e fauna. Holos, Ribeirão Preto, BR, p.257-277.

Meyers, N.; Mittermeier, R. A.; Mittermeier, C. G.; Fonseca, G. A. B.; Kent, J. 2000. Biodiversity hotspots for conservation priorities.
Nature, 403: 853-858.

Mikich, S. B.; Bérnils, R. S. 2004. Livro vermelho da fauna ameaçada no estado do Paraná. $16^{\mathrm{a}}$ ed. Instituto Ambiental do Paraná, Curitiba, BR, 764pp.

Miranda, D. B.; Garey, M. V.; Monteiro-Filho, E. L. A.; Hartmann, M. T. 2008. Sinalização visual e biologia reprodutiva de Dendropsophus werneri (Anura: Hylidae) em área de Mata Atlântica no Estado do Paraná, Brasil. Papéis Avulsos de Zoologia, 48 (29): 335-343.

Pimenta, B. V. S.; Haddad, C. F. B.; Nascimento, L. B.; Cruz, C. A. G.; Pombal Jr., J. P. 2005. Comment on "status and trends of amphibian declines and extinctions worldwide”. Science, 309 (23): 1999.

Pombal Jr., J. P. 1997. Distribuição espacial e temporal de anuros (Amphibia) em uma poça permanente na serra de Paranapiacaba, sudeste do Brasil. Revista Brasileira de Biologia, 57 (4): 583-594.

Pombal Jr., J. P.; Gordo, M. 2004. Anfíbios anuros da Juréia. In: Marques, O. A. V. \& Duleba, W. (Eds). Estação Ecológica Juréia Itatins: Ambiente físico, flora e fauna. Holos, Ribeirão Preto, BR, p.243-256.

Ribeiro, L. F.; Alvez, A. C. R.; Haddad, C. F. B.; Reis, S. F. 2005. Two new species of Brachycephalus Günther, 1858 from the state of Paraná, Southern Brazil (Amphibia, Anura, Brachycephalidae). Boletim do Museu Nacional, Zoologia, 519: 1-18.

Roderjan, C. V.; Galvão, F.; Kuniyoshi, Y. S.; Hatschbach, G. G. 2002. As unidades fitogeográficas do estado do Paraná, Brasil. Revista \& Ambiente, 24: 78-118.

Salthe, S. N.; Duellman, W. E. 1973. Quantitative constraints associated with reproductive mode in anurans. In: Vial, J. L. (Ed.). Evolutionary biology of the anurans. University of Missouri Press, Columbia, USA, p.229-249.

Scott Jr., N. J.; Woodward, B. D. 1994. Surveys at breeding sites. In: Heyer, W. R.; Donnelly, M. A.; McDiarmid, R. W.; Hayek, L. A. C. \& Foster, M. S. (Eds). Measuring and monitoring biological diversity - Standard methods for amphibians. Smithsonian Institution Press, Washington D. C., USA, p.84-92.

Segalla, M. V.; Langone, J. A. 2004. Anfíbios. In: Mikich, S. B. \& Bérnils, R. S. (Eds). Livro vermelho da fauna ameaçada no estado do Paraná. Instituto Ambiental do Paraná, Curitiba, BR, p. 537-577.

Silva, F. R.; Rossa-Feres, D. C. 2007. Uso de fragmentos por anuros (Amphibia) de área aberta na região noroeste do estado de São Paulo. Biota Neotropica 7 (2): 141-148.

Silvano, D. L.; Segalla, M. V. 2005. Conservação de anfíbios no Brasil. Megadiversidade, 1 (1): 70-86.

Sinsch, U.; Juraske, N. 2006. Advertisement calls of hemiphractine marsupial frogs: III Flectonotus spp. Proceedings of the $\mathbf{1 3}^{\text {th }}$ Congress of Societas Europaea Herpetologica, Bonn, Germany, p.153-157.

Steinbach-Padilha, G. C. 2008. A new species of Melanophryniscus (Anura, Bufonidae) from the Campos Gerais region of Southern Brazil. Phyllomedusa, 7 (2): 99-108.

Zimmerman, B. L.; Simberloff, D. 1996. An historical interpretation of habitat use by frogs in central Amazonian forest. Journal of Biogeography, 23 (1): 27-46.

Williams, S. E; Hero, J. M. 2001. Multiple determinants of Australian tropical frog biodiversity. Biological Conservation, 98: $1-10$. 